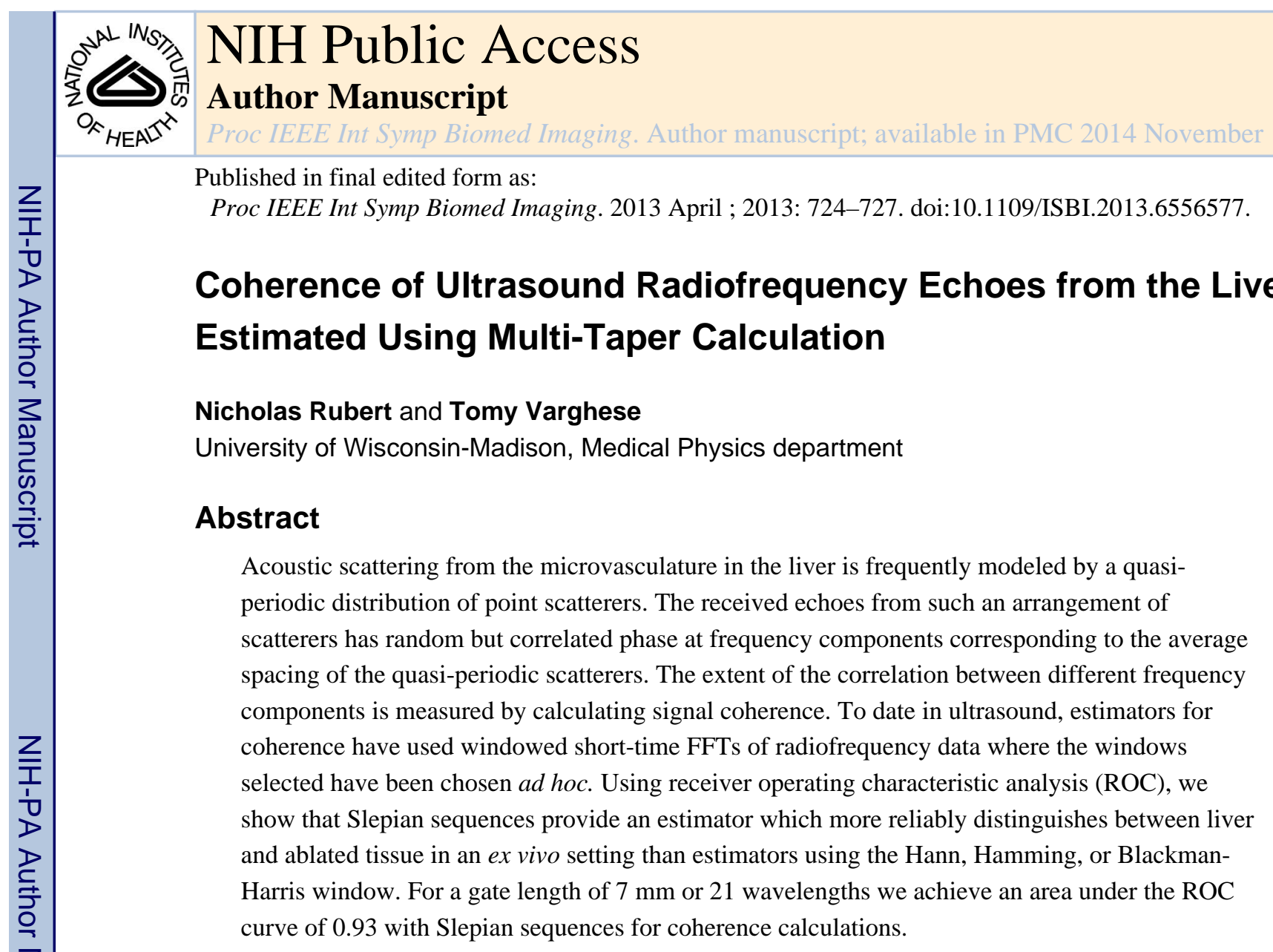

\title{
Index Terms
}

ultrasound; quantitative ultrasound; coherence

\section{Introduction}

It has been shown that a quasi-periodic arrangement of point-like scatterers is an accurate model for acoustic scattering in the liver [1], [2]. These scatterers are hypothesized to correspond to the microvasculature, and their mean spacing has been hypothesized to be an useful biomarker for pathological changes to the liver such as cirrhosis [1],[2]. Frequency domain techniques are commonly employed to estimate the spacing of quasi-periodic scatterers. In the pioneering work of Fellingham and Sommer [1], it was demonstrated that for a regular array of scatterers with a separation of $d$, peaks in the power spectrum would occur at frequency intervals, $\Delta f=c / 2 d$, where $c$ is the sound speed of the medium [1]. By measuring peaks in the autocorrelation of the power spectrum, Fellingham and Sommer were able to measure the spacing of peaks in the power spectrum and estimate mean scatterer spacing (MSS) measurements in the livers of human subjects [1]

The generalized spectrum (GS), referred to as spectral autocorrelation initially, was later introduced by Varghese and Donohue in [3] as a quantitative ultrasound (QUS) tool in order to quantify the MSS. Initially, Varghese and Donohue utilized a Welch-Bartlett average to estimate coherence[3]. They later demonstrated that due to the structure of the GS for 
signals from quasi-periodic scatterers a diagonally smoothed estimate of coherence may be more appropriate [4].

It has been well documented that thermally ablated liver is stiffer than liver tissue, and elasticity imaging has been shown to accurately delineate the extent of the thermal ablation[5], [6]. In this work, we demonstrate that echoes from ablated tissue also exhibit a decreased coherent component relative to echoes from the liver. We also show that a multitaper (MT) estimate of coherence is better able to distinguish between ablated liver tissue and normal liver tissue based on the presence or absence of coherent scattering using ROC analysis in an ex vivo setting.

\section{Theory}

Radiofrequency data from quasi-periodic scatterers may be modeled as zero mean nonstationary time series with correlated frequency components. This is a type of harmonizable process [7]. For a zero-mean, harmonizable process, $\mathrm{X}(\mathrm{t})$, the second-order behavior may be specified in terms of the autocovariance function or through the Loève spectrum. The two are related according to:

$$
\Gamma_{L}\left(t_{1}, t_{2}\right)=E\left[X\left(t_{1}\right) X^{*}\left(t_{2}\right)\right]=\int_{-\infty}^{\infty} \exp \left(i 2 \pi\left(t_{1} f_{1}-t_{2} f_{2}\right)\right) \gamma_{L}\left(f_{1}, f_{2}\right) d f_{1} d f_{2}
$$

The Loève spectrum depends on the spatial arrangement of the scatterers, overlying tissue attenuation, diffraction, and transducer bandwidth [8]. It has been shown that measuring coherence, as opposed to the GS, approximately deconvolves the effects of overlying tissue attenuation, diffraction, and transducer bandwidth [8]. Coherence is defined according to:

$$
C\left(f_{1}, f_{2}\right)=\frac{\gamma_{L}\left(f_{1}, f_{2}\right)}{\sqrt{\gamma_{L}\left(f_{1}, f_{1}\right) \gamma_{L}\left(f_{2}, f_{2}\right)}}
$$

Coherence takes on values from 0 to 1 , and is like a normalized cross-correlation function in this respect.

A diagonally smoothed estimate for the GS and the coherence has been proposed by Varghese and Donohue [4]. Diagonal smoothing is used because the Loève spectrum is expected to be continuous along diagonal lines corresponding to a constant scatterer spacing and discontinuous along lines perpendicular to this. Lines of constant scatterer spacing correspond to lines of constant frequency difference, $\mathrm{f}_{1}-\mathrm{f}_{2}=\mathrm{k}$ A diagonally smoothed Loève spectrum estimate over $\mathrm{N}_{\mathrm{A}}$ A-lines is given by:

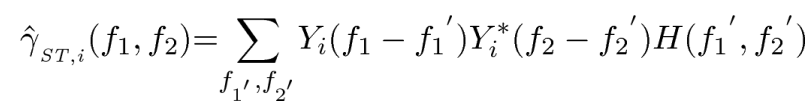




$$
\hat{\gamma}_{S T}\left(f_{1}, f_{2}\right)=\frac{1}{N} \sum_{i=1}^{N_{A}} \hat{\gamma}_{S T, i}\left(f_{1}, f_{2}\right) \phi\left(f_{1}\right) \phi^{*}\left(f_{2}\right)
$$

The $Y_{i}$ in the above equation refer to windowed DFTs of RF A-lines. In the equation above, $H$ is a smoothing kernel, and $i$ indexes individual A-lines. Diagonal smoothing is implemented by allowing $H\left(f_{1}, f_{2}\right)=\delta\left(f 1-f_{2}\right)$. The $\phi_{i}(f)$ are phase factors that account for the fact that the location of the first periodic scatterer may not be identical from one A-line to the next, and this produces phase offsets.

An MT estimate for the Loève spectrum has been employed in the field of geophysics [9]. The estimate of the Loève spectrum for the $\mathrm{i}^{\text {th }} \mathrm{A}$-line is given by:

$$
\begin{gathered}
\hat{\gamma}_{M T, i}\left(f_{1}, f_{2}\right)=\frac{A}{K} \frac{\sum_{k} \lambda_{k} d_{k}\left(f_{1}\right) y_{k}\left(f_{1}\right) d_{k}\left(f_{2}\right) y_{k}^{*}\left(f_{2}\right)}{\sqrt{\sum_{k} d_{k}\left(f_{1}\right)} \sqrt{\sum_{k} d_{k}\left(f_{2}\right)}} \\
A=\sum_{k=0}^{K-1} \frac{1}{\lambda_{k}}
\end{gathered}
$$

In the equation above, $K$ denotes the number of tapers used in the estimate, $\lambda_{\mathrm{k}}$ is the eigenvalue corresponding to the $k^{\text {th }}$ Slepian sequence, and $d_{k}$ are a set of data-adaptive weights derived by Thomson in his seminal paper on stationary power spectrum estimation[10]. Coherence is estimated by dividing an estimated Loève spectrum for a given A-line by its magnitude prior to averaging:

$$
\hat{C}\left(f_{1}, f_{2}\right)=\frac{1}{N_{A}} \sum_{i=1}^{N_{A}} \frac{\hat{\gamma}_{i}\left(f_{1}, f_{2}\right)}{\left|\hat{\gamma}_{i}\left(f_{1}, f_{2}\right)\right|}
$$

In the equation above, $\gamma_{\mathrm{i}}$ could be a diagonally smoothed single-taper (ST) or MT estimate of the Loève spectrum. In prior works this has been referred to as a system-normalized GS. In addition to coherence, the collapsed average (CA) is frequently calculated. The CA is an average along diagonal lines in the stationary direction. It is given by:

$$
\hat{\gamma}^{c}(\Delta f)=\frac{1}{M(g)} \sum_{f_{1}-f_{2}=g}\left|\hat{C}\left(f_{1}, f_{2}\right)\right|
$$

In the equation above, $M$ is a normalization constant that is equal to the number of discrete entries in a diagonal band corresponding to a single frequency difference. 


\section{Materials and Methods}

Four bovine livers were acquired from a slaughterhouse and 19 samples were excised. All procedures were performed within three days of acquiring the bovine liver, and all radiofrequency data sets were acquired using a clinical linear array transducer, the 9L4, on the Siemens S2000 scanner, at a transmit center frequency of $6 \mathrm{MHz}$.

For initial imaging, a sample was placed on a holder and immersed in physiological saline solution, and degassed in a vacuum chamber for 15 minutes. After degassing, the tissue was placed in a water bath maintained at a constant temperature of $37^{\circ} \mathrm{C}$ for 30 minutes. A radiofrequency data set of the liver was then acquired. The holder consisted of an L-shaped piece of plexiglass with two metal rods extending vertically from the leg of the " $\mathrm{L}$ ". The holder also had two holes drilled in its side which allowed for the insertion of metal fiducial rods through a specimen, to ensure imaging of similar planes. The bottom of the holder held a piece of rubber to avoid reverberations.

Following initial imaging of a sample, a radiofrequency (RF) ablated region was created in the imaging plane using an internally cooled CoolTip electrode with a $1 \mathrm{~cm}$ active region powered by a ValleyLab CoolTip RF generator (Radionics, Massachusetts, USA). For each ablation, the power was set to $12 \mathrm{~W}$ for 8 minutes in a manual mode. These settings produced ablations that were roughly spherical and approximately $1-1.5 \mathrm{~cm}$ in diameter. Following ablation, the RF applicator was removed, tissue was placed in physiological saline solution, degassed in a vacuum chamber for 15 minutes, and then placed in a water bath maintained at $37^{\circ} \mathrm{C}$ for 30 minutes. Another radiofrequency data set was then acquired. For the classification task this yielded 38 independent radiofrequency data sets. Nineteen data sets were of the liver prior to RF ablation, and 19 were of identical imaging planes in the same sample of liver following ablation. One pair of ablated and unablated data sets were acquired from each bovine liver sample.

Identical imaging settings were used for each pair of data sets collected. In all data sets, the time-gain curve (TGC) was uniformly set to the minimum setting across the whole imaging depth. Across all samples, the focal depth was set to $1 \mathrm{~cm}$. This depth was slightly shallower than the proximal end of the ablation being imaged. The presence of the RF ablated region in the imaging plane was confirmed using acoustic radiation force imaging (ARFI), available on the ultrasound system. A B-mode image with ROI selected prior to and following ablation is shown below in Fig. 1. All ROI's used in this analysis were $7 \mathrm{~mm}$ axially by $5 \mathrm{~mm}$ laterally. The staggered bright streaks in the image are the metal fiducial rods described earlier.

\section{Results}

We first show the coherence calculated in the ROI in Fig. 1. For all coherence calculations shown a smoothing radius of $0.642 \mathrm{MHz}$ was used for all ST calculations. An identical timebandwidth product, $\mathrm{NW}=0.642 \mathrm{MHz}$, was used for the MT calculations. Comparing the speckle pattern displayed in the B-mode images we notice that individual bright points are visible in the ROI prior to ablation. Following ablation, the ROI takes on a homogeneous appearance. We hypothesize that the ablation process results in a destruction of the 
scatterers that create coherent radiofrequency echo signals. This is reflected in the coherence calculations shown in Fig. 2. Whether using the MT method or a ST method of calculation we find that for the ROIs shown in Fig. 2 the region containing the isolated bright points prior to ablation shows a larger value for coherence than the region showing a homogeneous speckle pattern following ablation. Comparing the ST and MT calculation in Fig. 2, the coherence is elevated for the MT calculation when compared to the ST calculation.

We found that, in general, ROIs in the liver had an elevated value of coherence prior to ablation when compared to the same ROI following ablation. This was confirmed by constructing a tissue classifier. Tissue classifiers were constructed by first estimating the coherence in each of the 38 ROIs using the MT estimate and ST estimates with Hann, Hamming, and Blackman-Harris windows.

Following the coherence calculation, the mean about each ROI's center frequency was calculated prior to ablation, $C_{\text {pre }}\left(f_{c}+f_{1}, f_{c}+f_{2}\right)$, and following ablation, $C_{\text {post }}\left(f_{c}+f_{1}, f_{c}+f_{2}\right)$. The sample standard deviation was then computed in each frequency bin prior to, $\sigma_{\text {pre }}$ $\left(f_{c}+f_{1}, f_{c}+f_{2}\right)$, and following ablation, $\sigma_{\text {post }}\left(f_{c}+f_{1}, f_{c}+f_{2}\right)$. Due to changes in the attenuation of the signal following ablation the mean center frequency of signals following ablation was slightly lower than the center frequency of echo signals prior to ablation. Prior to ablation, a center frequency of $4.87 \pm 0.34 \mathrm{MHz}$ was measured and following ablation a center frequency of $4.62 \pm 0.17 \mathrm{MHz}$ was measured. After computing means and standard deviations, a tissue classifier was constructed using Fisher's linear discriminant:

$$
T=\frac{\gamma_{\text {pre }}^{c}-\gamma_{\text {post }}^{c}}{\sigma_{\text {pre }}+\sigma_{\text {post }}}
$$

Tissue templates for the Hann window and the MT calculation are shown below in Fig. 3. The templates are mostly positive outside the diagonal corresponding to the power spectrum, indicating that the coherence was larger on the average prior to ablation than following ablation.

Finally, each ROI was assigned a score by multiplying the template element-wise by the coherence calculated in the ROI and summing up the contributions from each discrete point in the bi-frequency plane. The ROC curve was then generated by varying a threshold. A false positive was an ablated ROI exhibiting a large mean coherence and a score above the threshold while a true positive was a region in the liver exhibiting a large mean coherence and a score above the threshold. The ROC analysis shows that the both MT calculation and ST calculations were able to consistently identify an ROI as being ablated based on a decrease in the signal coherence. However, the MT calculation outperformed the ST calculation, on the basis of the area under the ROC curve. The area under each ROC curve is summarized in Table 1. This is what is expected if the scattering in the ablated tissue is better modeled as diffuse than scattering in the liver, based on the superior leakage reduction properties of the Slepian sequences. 


\section{Conclusion}

In this work we have shown that the echoes from thermally ablated liver exhibit decreased coherence relative to echoes from the same liver prior to ablation. Future work will investigate whether this remains true at other transmit frequencies, and what effect the center frequency has on the sensitivity and specificity of the technique. Future work will also investigate whether coherence imaging could be used to delineate the extent of thermal ablation along with elasticity imaging. Improved coherence estimation may also be useful in classifying diffuse liver diseases.

\section{Acknowledgments}

This work was supported by NIH-NCI grants R01CA112192-S103, R01CA112192-05 and T32 CA09206-32

\section{References}

1. Fellingham LL, Sommer FG. Ultrasonic characterization of tissue structure in the in vivo human liver and spleen. IEEE Trans Ultrason Ferroelectr Freq Control. 1984; 31(4):418-428.

2. Landini L, Verrazzani L. Spectral characterization of tissues microstructure by ultrasounds: a stochastic approach. IEEE Trans Ultrason Ferroelectr Freq Control. 1990; 37(5):448-456. [PubMed: 18285062]

3. Varghese T, Donohue KD. Characterization of tissue microstructure scatterer distribution with spectral correlation. Ultrason imaging. 1993; 15(3):238-254. [PubMed: 8879094]

4. Varghese T, Donohue KD. Estimating mean scatterer spacing with the frequency-smoothed spectral autocorrelation function. IEEE Trans Ultrason Ferroelectr Freq Control. 1995; 42(3):451-463.

5. Varghese T, Zagzebski JA, Lee FT. Elastographic imaging of thermal lesions in the liver in vivo following radiofrequency ablation: preliminary results. Ultrasound in medicine \& biology. 2002; 28(11-12):1467-1473. [PubMed: 12498942]

6. Fahey BJ, Nightingale KR, McAleavey SA, Palmeri ML, Wolf PD, Trahey GE. Acoustic radiation force impulse imaging of myocardial radiofrequency ablation: initial in vivo results. IEEE Trans Ultrason Ferroelectr Freq Control. 2005; 52(4):631-641. [PubMed: 16060512]

7. Gerr Neil L, Allen Jeffery C. The Generalised Spectrum and Spectral Coherence of a Harmonizable Time Series. Digital Signal Processing. 1994; 4(4):222-238.

8. Donohue KD, Forsberg F, Piccoli CV, Goldberg BB. Analysis and classification of tissue with scatterer structure templates. IEEE Trans Ultrason Ferroelectr Freq Control. 1999; 46(2):300-310. [PubMed: 18238426]

9. Mellors R, Vernon F, Thomson D. Detection of dispersive signals using multitaper dual-frequency coherence. Geophysical J Int. 1998; 135(1):146-154.

10. Thomson DJ. Spectrum estimation and harmonic analysis. Proc IEEE. 1982; 70(9):1055-1096. 


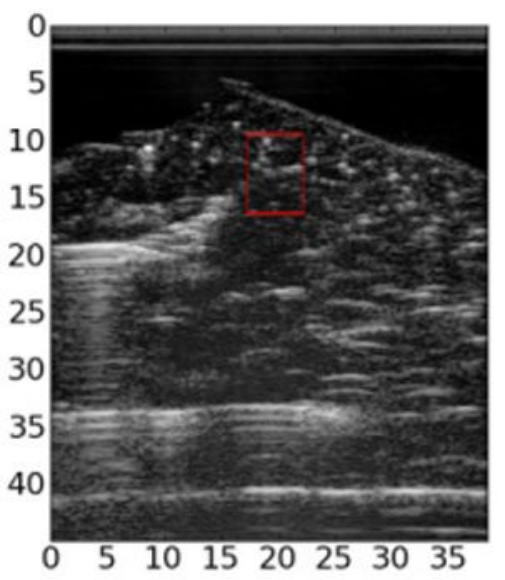

(A)

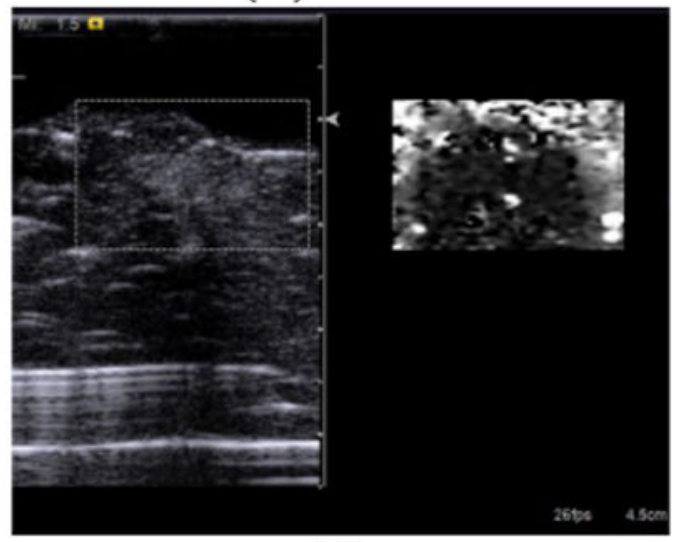

(C)

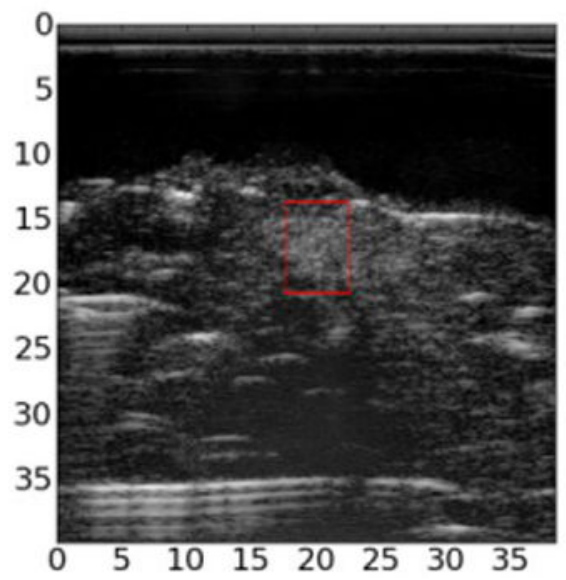

(B)

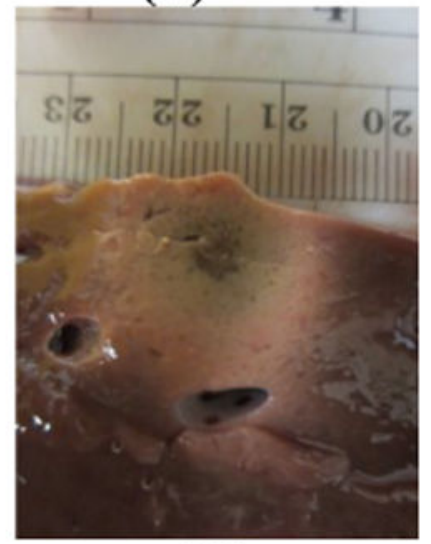

(D)

Fig. 1.

B-mode image prior to (A) and (B) following (B) RF ablation. (C) Clinical B-mode and ARFI image. (D) Optical pathology image. 


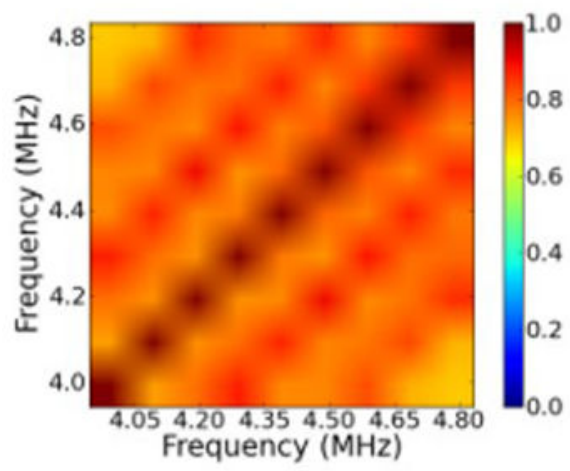

(A)

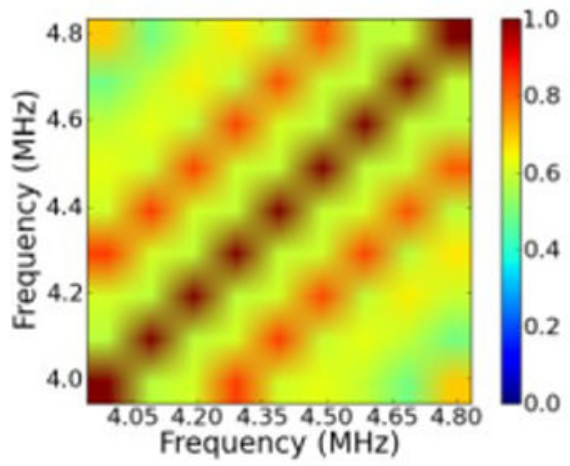

(C)

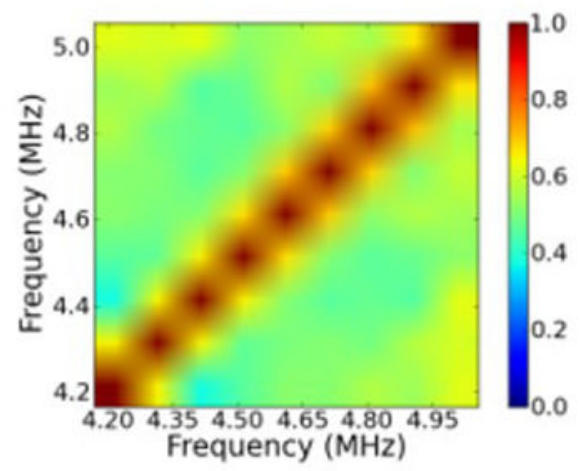

(B)

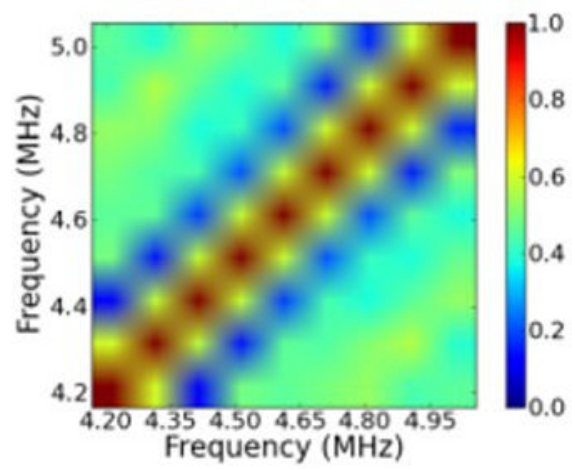

(D)

Fig. 2.

Coherence within the ROI in Fig. 1 calculated by a MT method prior to (A) and following (B) RF ablation. Coherence obtained using a ST method and a Hann window prior to (C) and following (D) ablation. 


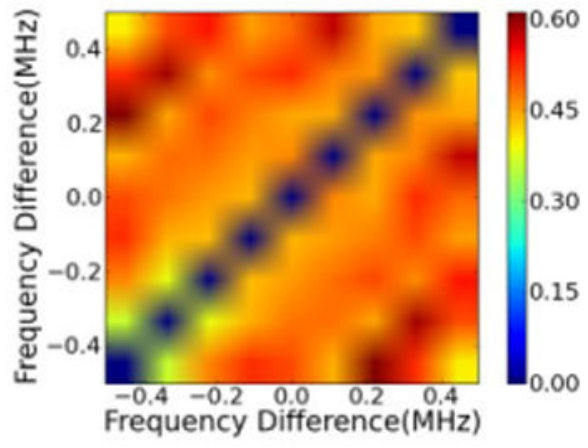

(A)

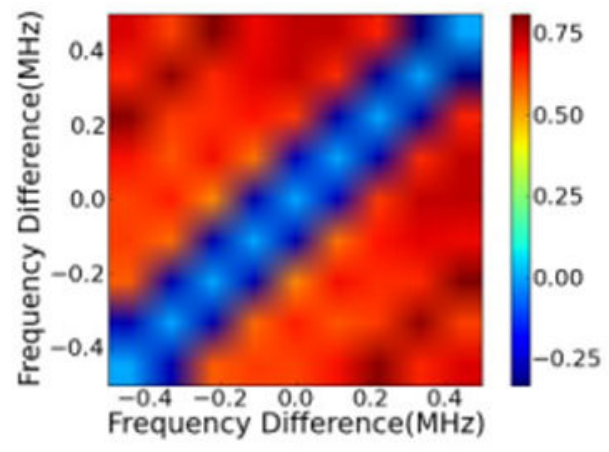

(B)

Fig. 3.

Templates created by (A) MT method (B) Hann window at a gate length of $5 \mathrm{~mm}$. 


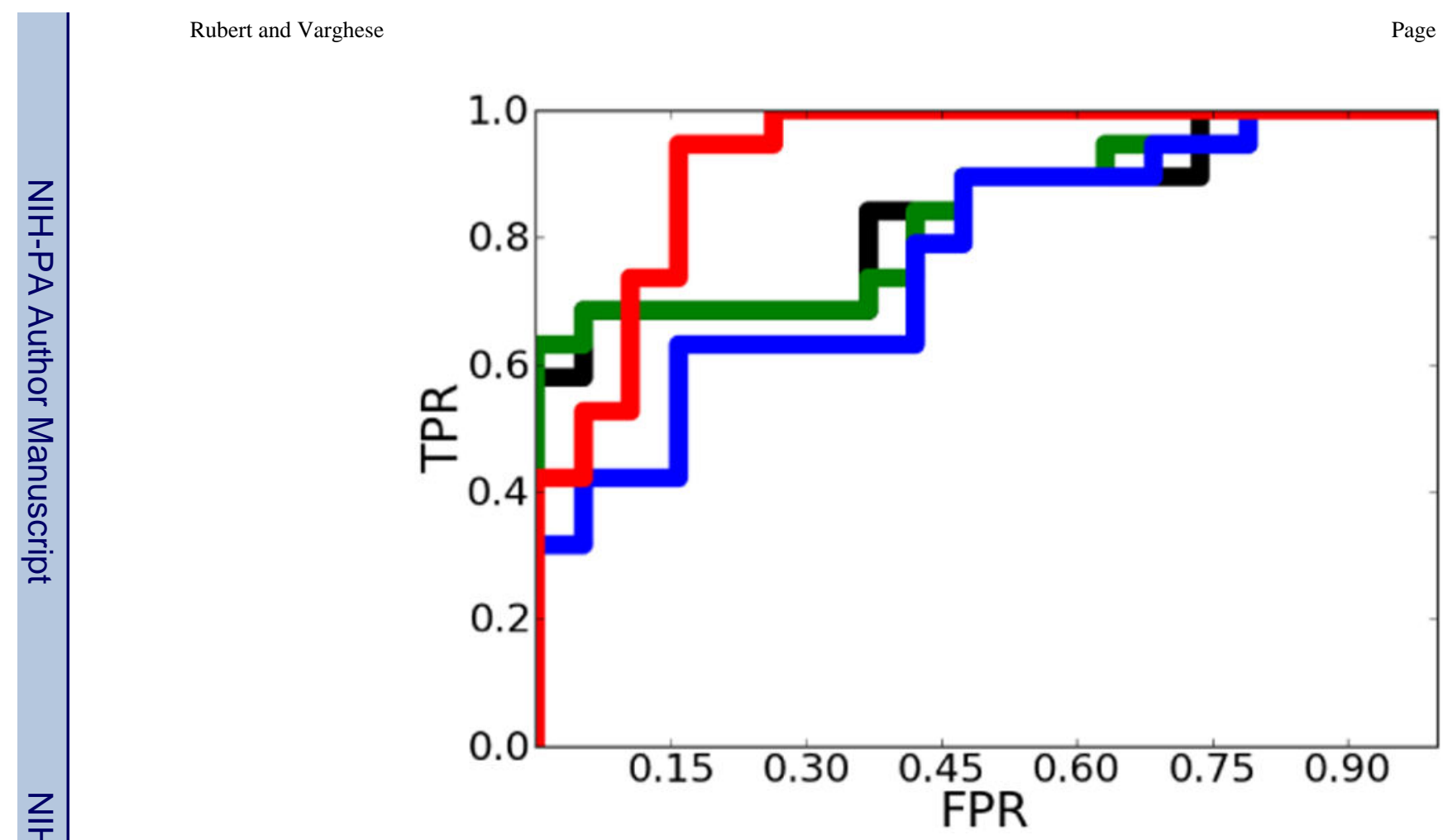

Fig. 4.

ROC curve. Red curve denotes MT calculation. Black curve denotes the Hann window. Green curve denotes Hamming window. Blue curve denotes Blackman-Harris window. 


\section{Table 1}

Area under ROC curves shown in Fig. 3.

\begin{tabular}{|c|c|c|c|c|}
\hline Method & MT & Hann & Hamming & BH \\
\hline Area & 0.93 & 0.83 & 0.83 & 0.77 \\
\hline
\end{tabular}

